\title{
THE EFFECTS OF CARVACROL ON OXIDATIVE STRESS PARAMETERS AND APOPTOSIS PROCESS VIA TRPA1 CHANNELS IN NEUROBLASTOMA CELLS
}

\author{
MEHMET YALCIN GUNAL* and ISHAK SUAT OVEY \\ Alanya Alaaddin Keykubat University, School of Medicine, \\ Department of Physiology, Antalya, Turkey
}

\begin{abstract}
Objectives: This study is a preliminary study to investigate the effects of carvacrol (CRV) obtained from thyme on the apoptosis process in neuroblastoma cells. Methods: In this study, seven groups were designed as control, CRV, CRV + AP-18, CRV + melatonin, CRV + melatonin + AP-18, melatonin and melatonin + AP-18. All groups were stimulated using CNM (cinnamaldehyde) which is TRPA1 channel stimulator. Levels of Reactive oxygen species (ROS), caspase-3, caspase-9, mitochondrial depolarization, apoptosis, intracellular free calcium and 3- (4,5-dimethylthiazol-2-yl) -2,5-diphenyltetrazolium bromide (MTT) were measured. Data were evaluated using one way ANOVA analysis. Results: Levels of ROS, mitochondrial depolarisation, caspase-3 and -9 and apoptosis were significantly higher in all groups treated with CRV compared to control $(\mathrm{p}<0.05)$. On the other hand, in melatonin-treated group and melatonin + AP-18 treated group, ROS and caspase- 3 were significantly lower than control $(\mathrm{p}<0.05)$. MTT levels were significantly decreased in all groups treated with CRV compared to control $(\mathrm{p}<0.05)$. On the other hand, in melatonin-treated group and melatonin + AP-18 treated group, MTT was higher than control $(\mathrm{p}<0.05)$. Conclusion: It has also been shown that CRV can also exert its effects through TRPA1 channels in neuroblastoma cells, which may accelerate the apoptosis process by acting on these channels, increasing ROS and caspase- 3 levels. Changes in MTT levels support this result. However, in order to better evaluate the effects of CRV on the apoptosis process, it would be useful to investigate changes in caspase- 9 , mitochondrial depolarization, and calcium channels.
\end{abstract}

Keywords: apoptosis, caspase-3, ROS, neuroblastoma, carvacrol

Transient receptor potential (TRP) family proteins are non-selective cation channels. TRP channels disentangle to seven separate sub-parent families: TRPA (Ankyrin), TRPC (Canonical), TRPM (Melastatin), TRPML (Mucolipins), TRPN (NOMPC), TRPP (Polycystin), and TRPV (Vanilloid). The sub-family members include six trans-membrane segments (1).

When the TRP channels activate, it depolarises the cell membrane, contributing in the activation or inactivation of voltage-gated ion channels, thus regulating the $\mathrm{Ca}^{2+}$ signal that controls various cellular functions (2, 3). TRPM2, TRPC5, TRPV1, and TRPA1 are as reactive oxygen-sensitive $\mathrm{Ca}^{2+}$-permeable channels. Reactive oxygen species activate TRPM2 (4-6). TRPC5, TRPV1 and TRPA1 channels reportedly play a role in the oxidative modification of cysteine residues when activated by reactive oxygen species $(7,8)$. Among these three TRP channels, TRPA1 has the maximum sensitiveness to reactive oxygen species. Therefore, it is TRPA1, not TRPC5 or TRPV1 which is thought to work as an oxidative stress receptor (9). Under various inflammatory diseases and pathological conditions, many cells manufacture large amounts of reactive oxygen species (ROS). TRP channels that are sensitive to ROS species may get activated and contribute to the exacerbation of various neurodegenerative diseases by causing changes in the $\mathrm{Ca}^{2+}$ flux $(10,11)$. Therefore, TRP channels that are activated by oxidative stress play a fundamental role in the pathophysiology of cancer and many neurodegenerative diseases $(1,3)$.

The activation of TRPA1 is triggered by cinnamon extracts, allyl isothiocyanate in mustard oil, and harmful cold impulse $(12,13)$; thus, TRPA1 also has a nociceptive function.

Neuroblastoma (NB) is the second uttermost prevalent extracranial malignant tumor in childhood (14). In spite of developments in treatment proto-

\footnotetext{
* Corresponding author: e-mail: yalcin.gunal@alanya.edu.tr; drmygunal@gmail.com
} 
cols, forward stage tumors are aggressive and commonly resist chemotherapeutic treatments. Recently studies have demonstrated that oxidative stress plays an essential role in the pathophysiology of neuroblastoma and many other neurodegenerative diseases, and the antioxidants compounds, such as plant extracts could prevent/reduce free radical damage in this diseases (15-19). Monoterpenes, which are present in numerous fruits, are known for their anti-oxidant properties. One of the monoterpenes obtained from essential oils of plants is carvacrol (CRV); it is a monoterpene (20-22) mainly found in thyme. Apart from its other effects, CRV has antioxidant properties (22-24) and is active on calcium-mediated TRPV1 and V3 channels in oral and nasal epithelial cells $(25,26)$. These studies contradict the results of a study by Zhen-wen et al. in 2015 that suggests that they are protective against the apoptosis of CRV on neuroblastoma cells (27). In this context, we aimed to demonstrate the effect of CRV on apoptosis through TRP channels in neuroblastoma cells.

\section{MATERIALS AND METHODS}

\section{Chemicals}

MTT (3-(4,5-Dimethylthiazol-2-yl)-2,5-Diphenyltetrazolium Bromide), a colorimetric assay method was purchased from Thermo Fischer (Waltham, MA, USA). Dihydrorhodamine-123 (DHR 123) was obtained from Molecular Probes (Eugene, OR, USA) and Dulbecco's modified Eagle's medium (DMEM), fetal bovine serum (FBS), trypsin-EDTA, dimethyl sulphoxide (DMSO), cinnamaldehyde, AP-18, penicillin-streptomycin, and carvacrol were purchased from Sigma-Aldrich Chemical (St. Louis, MO, USA). Fura-2/AM was obtained from Calbiochem (Darmstadt, Germany) and caspase 3 substrate (N-Acetyl-Asp-Glu-Val-Asp-7-amido-4-Methylcoumarin) (AC-DEVD-AMC) and caspase 9 substrate (Ac-Leu-Glu-His-Asp-7-Amino-4-methylcoumarin) (AC-LEHD-AMC) were purchased from Biovision (San Francisco, USA). APO Percentage dye with releasing buffer and a mitochondrial stain, 5,5',6,6'-tetrachloro-1,1',3,3'-tetraethylbenzimidazolylcarbocyanine iodide (JC-1), were provided from Biocolor (Belfast, Northern Ireland) and Santa Cruz (Dallas, Texas, USA), respectively.

\section{Experimental design}

The cinnamaldehyde (CNM; $0.1 \mathrm{mM}, 10 \mathrm{mM}$ ) was applied to the cells to activate the TRPA1 channel (28) (except for calcium signaling). During calcium signaling analysis (Fura-2/AM), cells were stimulated on $20^{\text {th }}$ cycles with $0.1 \mathrm{mM} \mathrm{CNM}$ in the presence of normal extracellular calcium $(1.2 \mathrm{mM})$. CNM and AP-18 were dissolved in DMSO, and the $\mathrm{pH}$ adjustment was carried out with $\mathrm{HCl}$.

All cells were incubated at $37^{\circ} \mathrm{C}$ and randomly divided into seven groups. In group 2, 3, 4 and 5, the dose of CRV was applied as $500 \mu \mathrm{M}$ and Mel was administered at $300 \mu \mathrm{M}$ dose in group 4, 5, 6 and 7 . Group 1 (Control): The cells were not incubated with CRV and melatonin (Mel) but were kept in a flask containing the same cell culture medium and conditions for $72 \mathrm{~h}$.

Group $2(\boldsymbol{C R V})$ : Cells in the group were incubated with CRV for $24 \mathrm{~h}(29)$.

Group $3(\boldsymbol{C R V}+\boldsymbol{A P}-18)$ : Cells in the group were incubated with CRV for $24 \mathrm{~h}$ and then incubated with the TRPA1 channel inhibitor AP-18 (0.02 mM, $30 \mathrm{~min})$.

Group $4(\boldsymbol{C R V}+\boldsymbol{M e l})$ : The cells in the group were incubated with CRV for $24 \mathrm{~h}$ and incubated with Mel for $2 \mathrm{~h}$.

Group $5(\boldsymbol{C R V}+\mathbf{M e l}+\boldsymbol{A P}-18)$ : Cells in the group were incubated with CRV for $24 \mathrm{~h}$, incubated with Mel for $2 \mathrm{~h}$ and then incubated with the TRPA1 channel inhibitor AP-18 (0.02 mM, $30 \mathrm{~min})$.

Group $6(\mathrm{Mel})$ : The cells in the group were incubated with Mel for $2 \mathrm{~h}$ (30).

Group $7(\mathbf{M e l}+\boldsymbol{A P}-\mathbf{1 8})$ : Cells in the group were incubated with Mel for $2 \mathrm{~h}$ and then incubated with the TRPA1 channel inhibitor AP-18 (0.02 mM, 30 $\min )$.

\section{Cell culture}

The SH-SY5Y human neuroblastoma cell line was obtained from ATCC (Manassas, VA). The cells were incubated in a cell culture medium containing Dulbecco's modified Eagle's medium (DMEM, Sigma Aldrich, St. Louis, MO) and HAM'S F12 (Biochrom, Berlin, Germany). This medium also contains 10\% FBS (Fisher Scientific) and $1 \%$ penicillin/streptomycin (Biochrom, Berlin, Germany). Cells were added in 8-10 flasks at a density of $1 \times 10^{6}$ cells per flask (filter cap, sterile, $5 \mathrm{~mL}, 25 \mathrm{~cm}^{2}$ ) and they were incubated in T25 flasks at $37^{\circ} \mathrm{C}$ at $5 \% \mathrm{CO}_{2}$ in a humidified incubator. After reaching $80 \%-85 \%$ confluence, the cells were washed with fresh $1 \times$ phosphate-buffered saline (PBS, Biochrom, Berlin, Germany), detached with $0.25 \%$ trypsin-EDTA (Sigma Aldrich, St. Louis, MO) and separated into the new culture flasks. After chemicals treatments corresponding to their groups, the cells were washed two times with PBS and allocated with $0.25 \%$ Trypsin-EDTA for analysis. CRV was dissolved in sterile distilled water as a 1-M 
stock solution. To investigate the effects of CRV on cytosolic calcium concentration, mitochondrial depolarization, ROS level, caspase-3 and -9 activations and apoptosis by TRPA1 channel activation,

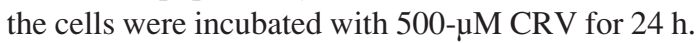
The other chemicals were dissolved in water, ethanol or DMSO. The concentration of organic solvents in the experimental solutions did not exceed $0.1 \%$ and did not affect the viability or basal intracellular free calcium concentration $\left[\mathrm{Ca}^{2+}\right]_{\mathrm{i}}$.

\section{Measurement of intracellular free calcium con- centration $\left(\left[\mathrm{Ca}^{2+}\right]_{\mathrm{i}}\right)$ \\ The $\left[\mathrm{Ca}^{2+}\right]_{\mathrm{i}}$ concentration and changes were} measured using UV light-excitable Fura-2 acetoxymethyl ester (Fura-2-AM) as an intracellular calcium ion indicator. The cells were isolated with $0.25 \%$ trypsin-EDTA after incubation with chemical compounds. Then, the cells were centrifuged for 5 min at $100 \mathrm{~g}$. The medium was removed and replaced with HEPES-buffered saline [HBS; 5-mM KCl, 145-mM NaCl, 10-mM D-glucose, 1-mM $\mathrm{MgCl}_{2}$, 1-mM $\mathrm{CaCl}_{2},(1.2 \mathrm{mM}), 10-\mathrm{mM}$ HEPES and $0.1 \%$ (w/v) bovine serum albumin (BSA); $\mathrm{pH} 7.4]$ containing 5- $\mathrm{\mu M}$ fura-2 $\mathrm{AM}$ and $0.05 \%(\mathrm{w} / \mathrm{v})$ pluronic F-127. After this step, the cells were incubated for $45 \mathrm{~min}$ at $37^{\circ} \mathrm{C}$ in the dark. The loaded cells were washed two times with HBS and covered with 1000 $\mu \mathrm{L}$ of HBS supplemented with $2.5-\mathrm{mM}$ probenecid for at least $20 \mathrm{~min}$ at $37^{\circ} \mathrm{C}$ in the dark. After Fura-2 AM esterification, the cells were placed in flat-bottom, black 96-well culture trays (Grainer Cell Star, Life Sciences USA) at a density of $2.5 \times 10^{4}$ cells/well. The fluorescence emission intensity of each well at $510 \mathrm{~nm}$ was measured using a plate reader $\left(\right.$ Synergy ${ }^{\mathrm{TM}} \mathrm{H} 1$, Biotek, USA). This procedure performed at alternating excitation wavelengths of 340 and $380 \mathrm{~nm}$ every $3 \mathrm{~s}$ for 50 acquisition cycles (cycle: 3 s; exposure: 25 flashes; gain: $120)$ in response to agonists (CNM, $0.1 \mathrm{mM}$ ). $\left[\mathrm{Ca}^{2+}\right]_{\mathrm{i}}$ in cells was expressed as the average emission at $510 \mathrm{~nm}$ in individual wells in response to the excitation at $340 / 380 \mathrm{~nm}$ normalized to initial fluorescence emission obtained during the first 10-20 cycles. The $\left[\mathrm{Ca}^{2+}\right]_{\mathrm{i}}$ was evaluated by a modified protocol (31).

\section{Intracellular reactive oxygen species measure- ment}

Dihydrorhodamine 123 (DHR 123) is a green fluorogenic probe that is used as a non-fluorescent reactive oxygen species (ROS) indicator and can pass through the cell membrane. DHR 123 becomes fluorescent upon oxidation to yield rhodamine 123
(Rh 123), with the fluorescence being proportional to ROS generation. DHR 123 can be readily insulated by active mitochondria and oxidized to rhodamine 123 . The cells $\left(10^{6}\right.$ cells $/ \mathrm{ml}$ for per group $)$ were incubated with $20 \mu \mathrm{m} \mathrm{DHR} 123$ at $37^{\circ} \mathrm{C}$ for 25 $\min (32)$. The $\mathrm{Rh} 123$ fluorescence intensities were measured using an automatic microplate reader (Synergy $^{\mathrm{TM}}$ H1, Biotek, USA). Excitation and emission wavelengths of the values were $488 \mathrm{~nm}$ and 543 $\mathrm{nm}$, respectively. The data were expressed as a fold increase compared to the pre-treatment level (experimental/control).

\section{Apoptosis assay}

The apoptosis assay was performed with a commercial kit by Biocolor Ltd. (Northern Ireland) according to the manufacturer's instructions (33). The detection of apoptosis by spectrophotometry was achieved at $550 \mathrm{~nm}$.

\section{Assay for caspase-3 and caspase-9 activities}

The activities of caspase- 3 and -9 were measured by the previously described method $(34,35)$. The cleavage of Caspase 3 substrate (N-Acetyl-AspGlu-Val-Asp-7-amido-4-Methylcoumarin) (ACDEVD-AMC) and caspase 9 substrate (Ac-Leu-GluHis-Asp-7-Amino-4-methylcoumarin) (AC-LEHDAMC) were determined with the microplate reader (Synergy $^{\mathrm{TM}}$ H1, Biotek, USA) with the excitation wavelength of $360 \mathrm{~nm}$ and an emission wavelength of $460 \mathrm{~nm}$. The values were expressed as fold increase over the pre-treatment level (experimental/control).

\section{Mitochondrial membrane potential (JC-1) ana- lyses}

The mitochondrial membrane potential was determined by a cationic fluorescent dye which can pass through the cell membrane. This dye accumulates in the normally respiring mitochondria and reduction the red-to-green fluorescence intensity in the medium shows mitochondrial depolarization. The membrane potential changes were evaluated by previously described methods (34). JC-1 fluorescence was measured by a single excitation wavelength $(488 \mathrm{~nm})$ with dual emission [green $(520 \mathrm{~nm})$ and red $(596 \mathrm{~nm})]$ using the microplate reader (Synergy ${ }^{\mathrm{TM}}$ H1, Biotek, USA). The dye concentrates in the mitochondrial matrix, wherein it creates red fluorescent aggregates (J-aggregates), and the dye is propagated throughout the entire cell leading to a shift from red (J-aggregates) to green fluorescence (JC-1 monomers). JC-1 values were assessed as fold increase relative to untreated control cells. 


\section{Cell viability (MTT) assay}

The MTT method for measuring the mitochondrial activity of living cells was used in this study to demonstrate the effects of Mel and CRV on cell viability. Cells were incubated with the chemical compounds described in the Experimental Design Section, fresh DMEM and HAM'S F12 medium containing MTT $(0.5 \mathrm{mg} / \mathrm{mL})$ were used to wash and incubate at $37^{\circ} \mathrm{C}$ for $90 \mathrm{~min}$ (33). DMSO was added to the supernatant to dissolve the formazan crystals and optical density was evaluated in an automatic microplate reader (Synergy ${ }^{\mathrm{TM}} \mathrm{H} 1$, Biotek, USA) (36). The cell viability assay was carried out 10 times. The data were given as fold increase by authors.

\section{Statistical analysis}

Differences between groups were analyzed by one-way ANOVA, followed by the least significant differences (LSD) tests. All values are given as mean \pm S.D. $p$ values $<0.05$ were considered statistically significant.

\section{RESULTS}

\section{Intracellular free calcium concentration $\left(\left[\mathrm{Ca}^{2+}\right]_{i}\right)$ measurement}

The calcium levels were higher in the CRV $(1.907 \pm 0.177), \mathrm{CRV}+\mathrm{AP}-18$ (1.533 \pm 0.245), $\mathrm{CRV}+\mathrm{Mel}(1.641 \pm 0.268), \mathrm{CRV}+\mathrm{Mel}+\mathrm{AP}-18$ $(1.299 \pm 0.222)$ groups compared to the control group, on the other hand lower in the Mel $(0.817 \pm$ $0.082)$ and Mel+AP-18 groups $(0.696 \pm 0.093$; $\mathrm{p}<$ 0.001 ; Figure 1A, B).

\section{Intracellular reactive oxygen species (ROS) meas- urement}

The ROS levels were higher in the CRV (2.289 \pm 0.018), CRV+AP-18 (2.010 \pm 0.007$), \mathrm{CRV}+\mathrm{Mel}$ $(2.062 \pm 0.036), \mathrm{CRV}+\mathrm{Mel}+\mathrm{AP}-18(1.992 \pm 0.005)$ groups compared to the control group, on the other hand lower in the Mel $(0.887 \pm 0.060)$ and Mel+AP18 groups $(0,856 \pm 0.009 ; \mathrm{p}<0.001$; Figure $1 \mathrm{C})$.

\section{Assay for caspase-9 and caspase-3 activities}

The caspase- 9 and caspase- 3 levels were higher in the CRV $(1.805 \pm 0,025 ; 1.470 \pm 0.011)$, CRV+AP-18 (1.575 $\pm 0.010 ; 1.378 \pm 0.015)$, CRV+Mel (1.610 $\pm 0.050 ; 1.257 \pm 0.015)$ CRV+Mel+AP-18 (1.321 $\pm 0.005 ; 1.149 \pm 0.014)$ groups compared to the control group, on the other hand lower in the Mel $(0.713 \pm 0.086)$ and $\mathrm{Mel}+\mathrm{AP}-$ 18 groups $(0.673 \pm 0.013$, respectively; $\mathrm{p}<0.001$; Figure 1D, E).

\section{Mitochondrial membrane potential (JC-1) analy-} ses

The mitochondrial depolarization levels were higher in the CRV $(1.523 \pm 0.011), \mathrm{CRV}+\mathrm{AP}-18$ (1.302 \pm 0.013), CRV+Mel (1.276 \pm 0.013), CRV+Mel+AP-18 (0.992 \pm 0.011$)$ groups compared to the control group, on the other hand lower in the $\mathrm{Mel}(0.866 \pm 0.034)$ and $\mathrm{Mel}+\mathrm{AP}-18$ groups $(0.778$ $\pm 0.026 ; \mathrm{p}<0.001 ;$ Figure $1 \mathrm{~F})$.

\section{Apoptosis assay}

The apoptosis levels were higher in the CRV $(1.492 \pm 0.016), \mathrm{CRV}+\mathrm{AP}-18(1.277 \pm 0.019)$, CRV+Mel (1.340 \pm 0.021), CRV+Mel+AP-18 $(1.212 \pm 0.021)$ groups compared to the control group, on the other hand lower in the Mel $(0.772 \pm$ $0.046)$ and Mel+AP-18 groups (0.741 $\pm 0.041 ; \mathrm{p}<$ 0.001; Figure 1G).

\section{Cell viability (MTT) assay}

The MTT levels were lower in the CRV (0.549 $\pm 0.004), \mathrm{CRV}+\mathrm{AP}-18(0.635 \pm 0.013), \mathrm{CRV}+\mathrm{Mel}$ $(0.718 \pm 0.011), \mathrm{CRV}+\mathrm{Mel}+\mathrm{AP}-18(0.820 \pm 0.010)$ groups compared to the control group, in contrast higher in the Mel $(1.350 \pm 0.040)$ and Mel+AP-18 groups $(1.393 \pm 0.030 ; \mathrm{p}<0.001 ;$ Figure $1 \mathrm{H})$.

\section{DISCUSSION}

Increases in the intracellular free calcium levels play a crucial role in events that initiate apoptosis at the cellular level. Increased calcium in the cell leads to an increase in ROS production. The increase in ROS levels leads to the activation of caspase- 9 by causing mitochondrial depolarization. Caspase-9 stimulates caspase-3, and apoptosis subsequently occurs (37-39).

CRV is a component of essential oils found in many plants. Numerous studies have reported on its varied biological effects. The effects of CRV on many tissues can be dose-dependent. Gunal et al. reported that the application of CRV at low doses to the skin has minimal effect on wound healing, whereas $12.5 \%$ concentration $(\mathrm{v} / \mathrm{v})$ showed optimal healing, and $=50 \%$ concentration $(\mathrm{v} / \mathrm{v})$ impaired the wound healing process (22). In a different study, Koparal and Zeytinoglu reported that CRV acts at different in NSCLC cells in a dose-dependent manner (29). The dose-dependent effects of CRV are similar to antioxidant properties. Studies have shown that low doses of CRV have antioxidant activity (40). Our study found that at higher doses, however, it triggers apoptosis via TRPA1 channel activation by increasing oxidative stress. 


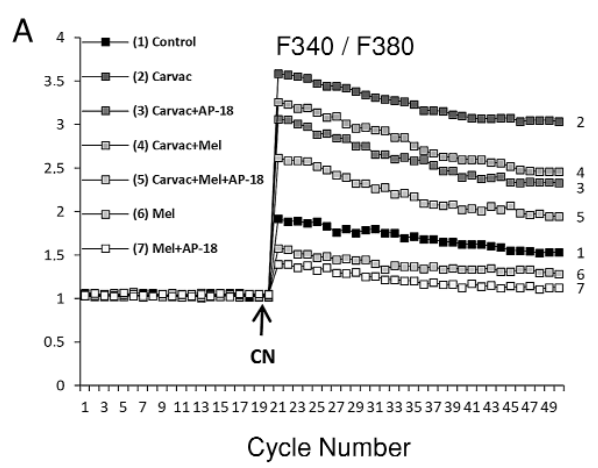

C

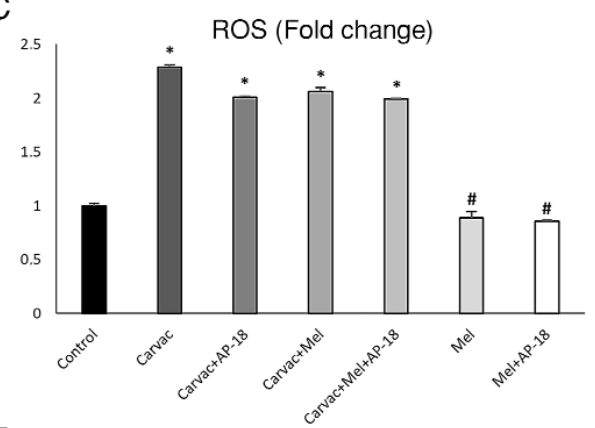

E

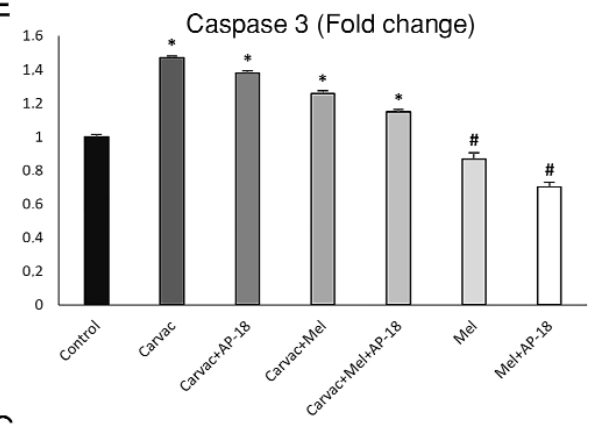

G

Apopitozis (Fold change)

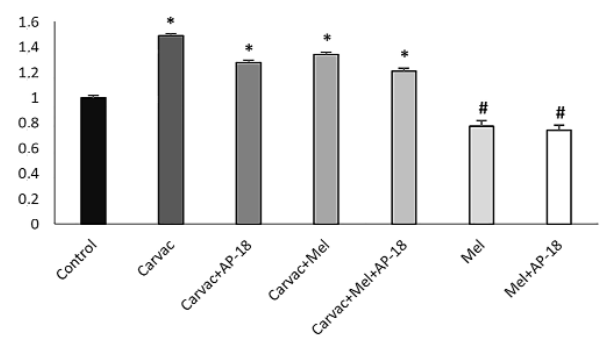

B

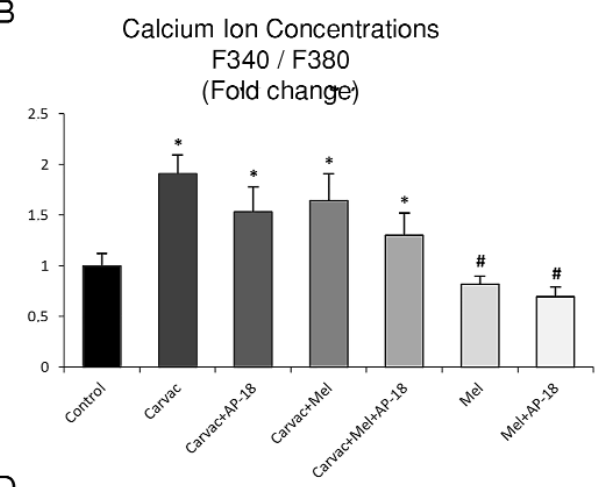

D

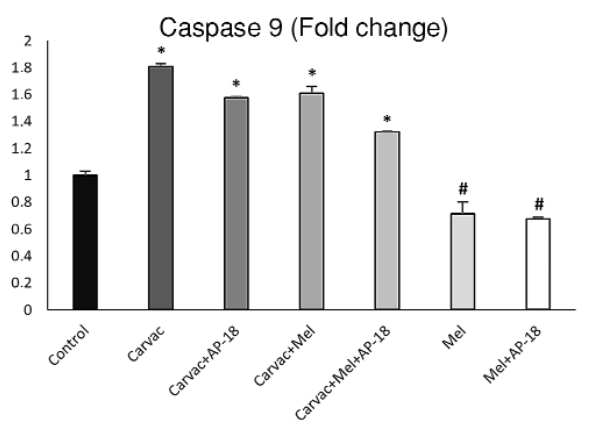

F

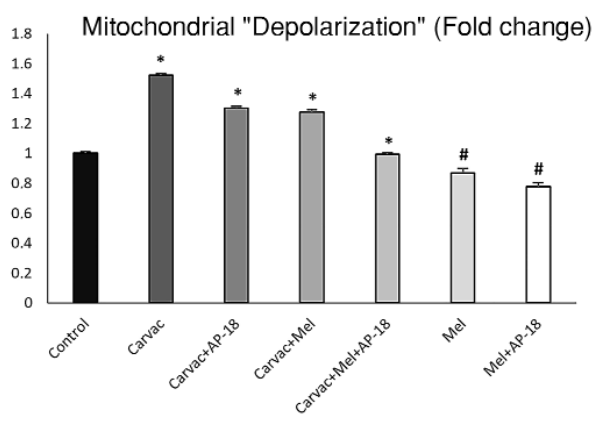

$\mathrm{H}$

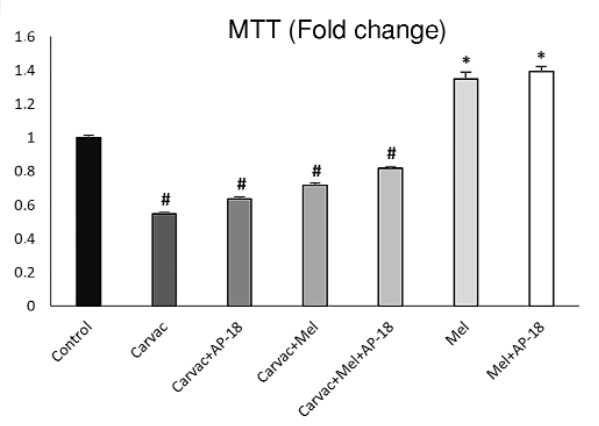

Figure 1. The effects of Carvacrol (500 $\mu \mathrm{M}, 24 \mathrm{hrs})$ and Melatonin ( $0,3 \mathrm{mM}, 2 \mathrm{hrs)}$ on Oxidative Stress Parameters and Apoptosis Process in SHSY-5Y cells. (A) Intracellular calcium levels. (B) Ca ion concentrations (fold change). (C) Reactive Oxygene Species (ROS) levels (fold change). (D) Caspase 9 levels (fold change). (E) Caspase 3 levels (fold change). (F) Mitochondrial Depolarization levels (fold change). (G) Apoptosis levels (fold change). (H) MTT (cell viability) levels (fold change). Calcium levels, ROS levels, mitochondrial depolarization levels, caspase-3 and -9 levels and apoptosis levels were statistically significantly higher in all CRV-treated groups than in the control group. Conversely, these parameters showed a statistically significant decrease in the Mel + AP-18 group compared to the control group. When MTT levels were evaluated, a statistically significant decrease was observed in all CRV-treated groups compared with the control group, whereas a significant increase was observed in the Mel-treated group and Mel + AP-18-treated group compared with the control group. * ${ }^{*}<0.001$ compared with the control group (indicates a statistically significant increase). ${ }^{*} \mathrm{p}<0.001$ compared with the control group (indicates a statistically significant decrease). All values are given as mean \pm S.D. 


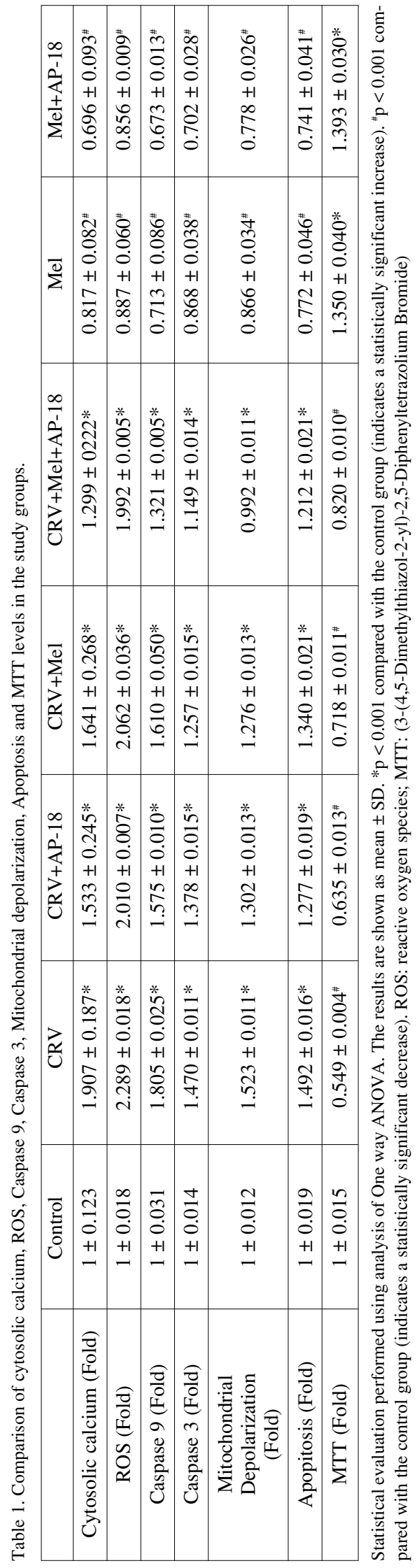

In our study, CNM was applied to all groups to activate TRPA 1 channels. The aim was to stimulate the passage of extracellular calcium into the cell by opening the channels. On the contrary, the AP-18 molecule used by us is a channel blocker that reduces the ion permeability of the channels by acting as an inhibitor on the same channels that CNM had a regulatory effect on. In the CRV-treated group, the calcium levels were increased, whereas in the CRV + AP-18-treated group, the calcium levels were significantly lower than they were in the CRV group; however, they did not descend to the control level (Figure 1A, B, p < 0.001). Taken together, we believe that CRV acts not only through TRPA1 channels but also through other ion channels. We have verified this situation with other parameters we have examined in our study.

$\mathrm{Mel}$ is a hormone known to have antioxidant and neuroprotective effects and is used as a reference molecule in many studies $(41,42)$. In our study, the application of Mel in the CRV-treated groups led to an antioxidant effect, resulting in a significant decrease in calcium levels and other apoptotic process parameters. However, the changes in these parameters did not reach the control group values in the CRV + Mel and CRV + Mel + AP18 groups. Therefore, the pro-oxidant effect of the CRV dose administered by us could not reach the control levels even with Mel. These findings reinforce our hypothesis that CRV has a potent apoptotic effect at the dose administered by us.

Zhen-wen et al. (2015) reported that CRV is protective against $\mathrm{Fe}$-induced apoptosis in neuroblastoma cells (27). On the contrary, the results of the present study indicate different effects. It has been reported that the CRV used in the study does not affect cell viability at a concentration of $333 \mu \mathrm{mol} / \mathrm{L}$ but is partially effective at a concentration of 666 $\mu \mathrm{mol} / \mathrm{L}$. In our study, on the contrary, it was found that CRV significantly decreased cell viability via TRPA1 channel in the TRPA1 channel inhibitor group compared to the control group (Figure 1H; $\mathrm{p}$ $<0.001)$. This finding is also consistent with intracellular calcium levels; calcium levels were found to be higher in the CRV group than in the control group (Figure 1A, B; p < 0.001). Given the mechanisms involved in the apoptosis process, it is expected that any agent that will increase apoptosis will cause an increase in mitochondrial depolarization and the levels of calcium, ROS and caspases 9 and 3. In the groups wherein CRV was applied, there was a statistically significant increase in the cytosolic calcium levels (Figure 1A, B; p < 0.001), ROS levels (Figure 1C; p < 0.001), caspase-9 levels 
(Figure 1D; $\mathrm{p}<0.001$ ), caspase-3 levels (Figure 1E; $\mathrm{p}<0.001$ ), mitochondrial depolarization (Figure 1F; $\mathrm{p}<0.001$ ) and apoptosis (Figure 1G; $\mathrm{p}<0.001$ ) in accordance with this reaction chain. In our study, it was determined that CRV decrease cell viability, which was in accordance with these results (Figure $1 \mathrm{H} ; \mathrm{p}<0.001)$.

\section{CONCLUSION}

In our study, 500- $\mu \mathrm{M}$ CRV has been shown to increase calcium levels in SH-SY5Y neuroblastoma cells, thus inducing apoptosis and increasing oxidative damage. In the light of these data, we conclude that CRV may be useful in the treatment of neuroblastoma and that more advanced studies exploring the effects of CRV are needed.

\section{Conflict of interest statement}

We declare that we have no conflict of interest.

\section{REFERENCES}

1. Vennekens R., Menigoz A., Nilius B.: Rev. Physiol. Biochem. Pharmacol. 163, 27 (2012).

2. Wu L.J., Sweet T.B., Clapham D.E.: Pharmacol. Rev. 62, 381 (2010).

3. Nilius B., Szallasi A.: Pharmacol. Rev. 66, 676 (2014).

4. Hara Y., Wakamori M., Ishii M., Maeno E., Nishida M. et al.: Mol. Cell 9, 163 (2002).

5. Ishii M., Shimizu S., Hara Y., Hagiwara T., Miyazaki A. et al.: Cell Calcium 39, 487 (2006).

6. Shimizu S., Yonezawa R., Negoro T., Yamamoto S., Numata T. et al.: Int. J. Biochem. Cell Biol. 68, 119 (2015).

7. Yoshida T., Inoue R., Morii T., Takahashi N., Yamamoto S. et al.: Nat. Chem. Biol. 2, 596 (2006).

8. Takahashi N., Mizuno Y., Kozai D., Yamamoto S., Kiyonaka S. et al.: Channels (Austin). 2, 287 (2008).

9. Yamamoto S., Shimizu S.: Eur. J. Pharmacol. 793, 109 (2016).

10. Droge W.: Physiol. Rev. 82, 47 (2002).

11. Lambeth J.D.: Nat. Rev. Immunol. 4, 181 (2004).

12. Story G.M., Peier A.M., Reeve A.J., Eid S.R., Mosbacher J. et al.: Cell. 112, 819 (2003).

13. Jordt S.E., Bautista D.M., Chuang H.H., McKemy D.D., Zygmunt P.M. et al.: Nature. 427, 260 (2004).
14. Betts D.R., Cohen N., Leibundgut K.E., Kuhne T., Caflisch U. et al.: Pediatr. Blood Cancer 44, 147 (2005).

15. Cadet J.L., Brannock C.: Neurochem. Int. 32, 117 (1998).

16. Halliwell B.: J. Neurochem. 97, 1634 (2006).

17. Turkez H., Togar B., Polat E.: Cytotechnology 64, 459 (2012).

18. Shon M.Y., Choi S.D., Kahng G.G., Nam S.H., Sung N.J.: Food Chem. Toxicol. 42, 659 (2004).

19. Dirican E., Turkez H., Togar B.: Cytotechnology 64, 181 (2012).

20. Zeytinoglu H., Incesu Z., Baser K.H.: Phytomedicine 10, 292 (2003).

21. Keskin I., Gunal Y., Ayla S., Kolbasi B., Sakul A. et al.: Biotech. Histochem. 92, 274 (2017).

22. Gunal M.Y., Heper A.O., Zaloglu N.: Pharmacognosy J. 6, 10 (2014).

23. Baser K.H.: Curr. Pharm. Des. 14, 3106 (2008).

24. Lagouri V., Boskou D.: Int. J. Food Sci. Nutr. 47, 493 (1996).

25. Vogt-Eisele A.K., Weber K., Sherkheli M.A., Vielhaber G., Panten J. et al.: Br. J. Pharmacol. 151, 530 (2007).

26. Xu H., Delling M., Jun J.C., Clapham D.E.: Nat. Neurosci. 9, 628 (2006).

27. Cui Z.W., Xie Z.X., Wang B.F., Zhong Z.H., Chen X.Y. et al.: Acta Pharmacol. Sin. 36, 1426 (2015).

28. Kimura Y., Mikami Y., Osumi K., Tsugane M., Oka J., Kimura H.: FASEB J. 27, 2451 (2013).

29. Koparal A.T., Zeytinoglu M.: Cytotechnology 43, 149 (2003).

30. Celik O., Naziroglu M.: Physiol. Behav. 107, 458 (2012).

31. Martinez N.A., Ayala A.M., Martinez M., Martinez-Rivera F.J., Miranda J.D., Silva W.I.: J. Biol. Chem. 291, 12208 (2016).

32. Espino J., Pariente J.A., Rodriguez A.B.: World J. Diabetes. 2, 82 (2011).

33. Ovey I.S., Naziroglu M.: Neuroscience 284, 225 (2015).

34. Bejarano I., Redondo P.C., Espino J., Rosado J.A., Paredes S.D. et al.: J. Pineal Research. 46, 392 (2009).

35. Espino J., Bejarano I., Redondo P.C., Rosado J.A., Barriga C. et al.: J. Membr. Biol. 233, 105 (2010).

36. Pariente R., Bejarano I., Rodriguez A.B., Pariente J.A., Espino J.: Mol. Cell Biochem. 440, 43 (2018).

37. Ghobrial I.M., Witzig T.E., Adjei A.A.: CA Cancer J. Clin. 55, 178 (2005). 
38. Repnik U., Stoka V., Turk V., Turk B.: Biochim. Biophys. Acta 1824, 22 (2012).

39. Ou L., Lin S., Song B., Liu J., Lai R., Shao L.: Int. J. Nanomedicine 12, 6633 (2017).

40. Undeger U., Basaran A., Degen G.H., Basaran N.: Food Chem. Toxicol. 47, 2037 (2009).
41. Kilic U., Caglayan A.B., Beker M.C., Gunal M.Y., Caglayan B. et al.: Redox Biol. 12, 657 (2017).

42. Deng S.L., Sun T.C., Yu K., Wang Z.P., Zhang B.L. et al.: Free Radic. Biol. Med. 113, 347 (2017).

Received: 16.08.2018 